\title{
Early Listening Function (ELF): adaptação para a língua portuguesa
}

\author{
Early Listening Function (ELF): adaptation into Brazilian \\ Portuguese
}

\author{
Marluci Oshima ${ }^{1}$, Adriane Lima Mortari Moret ${ }^{2}$, Raquel Beltrão Amorim ${ }^{3}$, Kátia de Freitas Alvarenga ${ }^{4}$, Maria Cecília \\ Bevilacqua $^{5}$, José Roberto Pereira Lauris ${ }^{6}$, Regina Tangerino de Souza Jacob ${ }^{7}$
}

\begin{abstract}
RESUMO
Objetivo: Traduzir e adaptar culturalmente para a língua portuguesa o questionário Early Listening Function (ELF), e avaliar a confiabilidade do mesmo. Métodos: Foi realizada a tradução do questionário para o idioma Português, revisão das equivalências gramatical e idiomática (traduções reversas) e adaptações linguística e cultural. Além disso, foi avaliada a reprodutibilidade intrapesquisadores. Após a tradução, o ELF foi aplicado, em ambiente silencioso e ruidoso, em 30 crianças entre zero e três anos de idade, sem histórico de risco para deficiência auditiva, ausência de queixa familiar quanto ao desenvolvimento global da criança e sem indicadores de perda auditiva incapacitante, em diferentes distâncias, em 12 situações de detecção auditiva. Resultados: Os resultados foram analisados com estudo estatístico descritivo a partir da pontuação obtida no ELF. Conclusão: O instrumento ELF foi traduzido e adaptado culturalmente para a população estudada. No Português, sua denominação manteve a sigla ELF. O estudo permitiu verificar sua confiabilidade para observação e acompanhamento das etapas iniciais do comportamento auditivo.
\end{abstract}

Descritores: Tradução (Processo); Transtornos da audição/diagnóstico; Testes auditivos; Questionários; Perda auditiva/diagnóstico; Diagnóstico precoce; Lactente; Desenvolvimento da linguagem

\section{INTRODUÇÃO}

O foco primário de um programa de intervenção precoce

Trabalho realizado no Departamento de Fonoaudiologia da Faculdade de Odontologia de Bauru da Universidade de São Paulo - USP - Bauru (SP), Brasil, com auxílio PIBIC/CNPQ. Trabalho apresentado parcialmente no $16^{\circ}$ Congresso de Fonoaudiologia (Campos do Jordão, 2008) e no Simpósio Internacional de Iniciação Cientifica da Universidade de São Paulo (Ribeirão Preto, 2008).

(1) Fonoaudióloga clínica - Bauru (SP), Brasil.

(2) Doutora, Professora do Departamento de Fonoaudiologia da Faculdade de Odontologia de Bauru da Universidade de São Paulo - USP - Bauru (SP), Brasil.

(3) Pós-graduanda (Mestrado) em Fonoaudiologia do Departamento de Fonoaudiologia da Faculdade de Odontologia de Bauru da Universidade de São Paulo - USP - Bauru (SP), Brasil.

(4) Doutora, Professora do Departamento de Fonoaudiologia da Faculdade de Odontologia de Bauru da Universidade de São Paulo - USP - Bauru (SP), Brasil.

(5) Professora Titular da Faculdade de Odontologia de Bauru da Universidade de São Paulo - USP - Bauru (SP), Brasil.

(6) Doutor, Professor Associado do Departamento de Odontopediatria, Ortodontia e Saúde Coletiva da Faculdade de Odontologia de Bauru da Universidade de São Paulo - USP - Bauru (SP), Brasil.

(7) Doutora, Professora do Departamento de Fonoaudiologia da Faculdade de Odontologia de Bauru da Universidade de São Paulo - USP - Bauru (SP), Brasil.

Endereço para correspondência: Regina Tangerino de Souza Jacob. Al. Dr. Otávio Pinheiro Brizola, 9-75, Bauru (SP), Brasil, CEP: 17012-901. E-mail: reginatangerino@usp.br

Recebido em: 3/5/2009; Aceito em: 26/10/2009 na deficiência auditiva é dar suporte e encorajar os familiares na estruturação do processo de comunicação da criança $a^{(1-3)}$. Estudos sobre a comunicação de crianças com deficiência auditiva têm mostrado que a participação da família no processo de intervenção é potencialmente um dos aspectos de maior impacto no desenvolvimento da linguagem oral, quando comparado com a idade de identificação da perda auditiva, a intervenção e o grau da perda ${ }^{(4)}$. Por outro lado, nem sempre os pais estão preparados para o diagnóstico da deficiência auditiva logo nos primeiros meses de vida da criança. Para muitos pais, as reações emocionais iniciais são passageiras e estabilizadoras, mas para outros podem perdurar por anos ou tornarem-se permanentes ${ }^{(5)}$. A literatura aponta e destaca a importância da família no processo de identificação( ${ }^{(6)} \mathrm{e}$ diagnóstico da deficiência auditiva e a contribuição da família no que se refere às observações de comportamentos e respostas sutis da criança em contextos construídos com esse objetivo ${ }^{(7,8)}$. O fato de a família acompanhar o processo de avaliação refletirá positivamente na compreensão e aceitação desta nova realidade $^{(6)}$. Os pais podem ser influenciados em suas atitudes pela época em que o diagnóstico foi definido e a maneira como o processo foi conduzido ${ }^{(9)}$.

O conhecimento do comportamento auditivo próprio da criança pode auxiliar a família a compreender a importância da proximidade e intensidade da fonte sonora na detecção dos sons de fala e ambientais, no desenvolvimento de outras habilidades auditivas mais complexas, no monitoramento de 
alterações, como, por exemplo, ocorrência de otites, flutuação e progressão da perda auditiva e ainda na identificação do funcionamento correto do dispositivo eletrônico adaptado(3).

Vários foram os testes clínicos desenvolvidos para a avaliação da percepção dos sons da fala em crianças pequenas ${ }^{(10-15)}$, no entanto, pouca atenção tem sido dedicada aos pais e familiares no processo de detecção precoce da deficiência auditiva ${ }^{(16)}$.

Neste contexto, foi criado o instrumento ELF - Early Listening Function ${ }^{(3,17)}$ especificamente para ser aplicado em casa pelos pais. O ELF é um instrumento que incentiva os pais a participarem do processo de avaliação. Os pais são encorajados a observar e registrar o comportamento auditivo da criança em situações domiciliares, sendo possível avaliar a capacidade da criança em detectar sons diversos e em diferentes posições.

Assim, este estudo teve como objetivo traduzir e adaptar culturalmente para a língua portuguesa o questionário ELF Early Listening Function e avaliar a confiabilidade do mesmo.

\section{MÉTODOS}

O ELF (Anexo 1) foi entregue para 75 pais/responsáveis de crianças entre cinco meses e três anos de idade. Concordaram com a participação nesta pesquisa as famílias de 30 crianças de ambos os sexos. Os respectivos responsáveis das trinta crianças assinaram o Termo de Consentimento Livre e Esclarecido, conforme modelo aprovado pela Comissão de Ética em Pesquisa da instituição sob protocolo número 058/2007, atestando sua permissão para a participação da criança no trabalho e publicação dos dados obtidos.

Como critério de inclusão, as crianças não poderiam apresentar histórico de risco para deficiência auditiva, queixa familiar quanto ao desenvolvimento global e indicadores de perda auditiva incapacitante ${ }^{(18)}$ verificados por meio de observação clínica comportamental. Os pais/responsáveis também responderam ao questionário para classificação econômica no Critério Brasil ${ }^{(19)}$.

\section{Instrumentos e procedimentos}

O ELF original ${ }^{(3,17)}$ contém 12 atividades de detecção auditiva para sons de fraca, média e forte intensidades, planejadas para serem apresentadas pelos pais de crianças a partir de cinco meses a três anos de idade, a diferentes distâncias medidas com uma trena ( $15 \mathrm{~cm}, 1 \mathrm{~m}, 2 \mathrm{~m}, 3 \mathrm{~m}$ e a aproximadamente $5 \mathrm{~m}$ ), em duas situações, ou seja, em ambiente silencioso e com ruído.

$\mathrm{O}$ instrumento oferece as opções de respostas $\operatorname{sim}(\mathrm{S})$, não $(\mathrm{N})$ e às vezes $(\mathrm{A})$ para cada reação da criança frente aos estímulos sonoros.

É possível pontuar o escore de cada aspecto, ou seja, a pontuação realizada nas atividades em volume fraco, em volume normal e em volume forte/alto, através da marcação de quantas atividades a criança respondeu (número de sim) e as atividades que a criança respondeu na metade das vezes (número de às vezes), as diferentes distâncias. Essa quantidade de respostas da criança é multiplicada e obtém-se um valor total, que deverá ser transferido para a outra tabela. Nesta tabela, é somada a pontuação total em cada aspecto (silêncio + ruído) nas diferentes situações. Desta forma, considera-se que o maior escore possível corresponda a 100 no total do silêncio e no total do ruído, oito para o número de sim nas atividades em volume fraco, assim como nas atividades em volume normal e em volume alto/forte e 200 para a pontuação total no silêncio + ruído.

\section{Adaptação cultural para a língua portuguesa}

A tradução e adaptação cultural do questionário $\operatorname{ELF}^{(3,17)}$ seguiram as seguintes etapas ${ }^{(20,21)}$ :

- Tradução do questionário para o idioma Português. $\mathrm{O}$ instrumento na versão original foi distribuído para dois tradutores-intérpretes de Inglês, fluentes nesse idioma, que não se conheciam e não conheciam o questionário, visando elaborar individual e sigilosamente a primeira versão para o Português. Este procedimento foi realizado com o intuito de gerar duas traduções independentes do ELF.

- Adaptação linguística. O grupo revisor foi constituído por duas fonoaudiólogas (brasileiras, fluentes na língua inglesa), que analisaram os dois documentos resultantes e reduziram as diferenças encontradas nas traduções, adaptando o texto à cultura brasileira. Desta forma, foi obtido um novo inventário denominado ELF.

- Revisão das equivalências gramatical e idiomática (traduções reversas). Para a revisão da equivalência gramatical e idiomática, uma cópia do ELF foi encaminhada para dois outros tradutores, de mesma condição linguística e cultural dos primeiros. Estes, desconhecedores do texto original, realizaram nova versão do instrumento para o idioma Inglês. O mesmo grupo revisor realizou nova avaliação das duas versões resultantes, comparando-as com a original em Inglês.

- Adaptação cultural. Tem como objetivo estabelecer a equivalência cultural entre as versões inglesa e portuguesa do questionário. A equivalência cultural é estabelecida se não forem observadas dificuldades de compreensão das questões elaboradas ou dos termos utilizados, por, no mínimo, $80 \%$ da população avaliada.

\section{Confiabilidade do questionário}

Ao considerar que todas as crianças apresentavam audição normal, esperou-se o mesmo padrão de respostas para as atividades apresentadas. Para verificar esta hipótese dividiu-se a casuística controlando as variáveis de nível econômico e grau de instrução do chefe da família. Para esta classificação utilizou-se o Critério de Classificação Econômica Brasil ${ }^{(19)}$, que foi construído para definir classes que atendam às necessidades de segmentação por poder aquisitivo, enfatizando o poder de compra das pessoas e famílias urbanas, abandonando a pretensão de classificar a população em termos de classes sociais.

\section{Método estatístico}

Para a análise estatística foi utilizado o Teste $\mathrm{t}$ com o intuito de avaliar a diferença quanto aos resultados obtidos nos escores do questionário ELF, intra-pesquisadores. Neste estudo foi utilizado nível de significância $\mathrm{p} \leq 0,05$. 
Tabela 1. Análise descritiva dos escores obtidos no ELF aplicado pelos pais/responsáveis

\begin{tabular}{|c|c|c|c|c|c|}
\hline \multirow[t]{2}{*}{ Variação } & \multicolumn{2}{|c|}{ Classe A/B } & \multicolumn{2}{|c|}{ Classe C/D } & \multirow[t]{2}{*}{ Valor de $\mathrm{p}$} \\
\hline & Média & DP & Média & DP & \\
\hline Total no silêncio & 87,23 & 7,47 & 89,76 & 12,30 & 0,51 \\
\hline Total no ruído & 79,71 & 9,22 & 85,33 & 11,81 & 0,16 \\
\hline Total silêncio+ruído & 166,64 & 15,01 & 175,01 & 21,70 & 0,23 \\
\hline Número de sim nas atividades em volume fraco & 5,81 & 1,02 & 6,33 & 1,51 & 0,29 \\
\hline Número de sim nas atividades em volume normal & 6,77 & 0,78 & 7,50 & 0,62 & $0,01^{*}$ \\
\hline Número de sim nas atividades em volume forte/alto & 7,91 & 0,23 & 7,84 & 0,33 & 0,47 \\
\hline
\end{tabular}

Teste $t$. *Diferença estatisticamente significante $(p \leq 0,05)$

Legenda: DP = desvio-padrão

\section{RESULTADOS}

Nesta pesquisa, 30 crianças sem indicadores de perda auditiva incapacitante (de acordo com os critérios da $\mathrm{WHO}^{(18)}$ participaram do processo de tradução e adaptação cultural do ELF. Destas, 11 eram do sexo feminino $(37,7 \%)$ e 19 eram do sexo masculino (63,3\%), com idade entre dez meses e três anos (média de 22,9 meses).

O ELF, obtido após a tradução do idioma Inglês para o Português e a adaptação linguística, encontra-se no Anexo 1 , e as folhas de pontuação para análise do escore de cada aspecto no Anexo 2.

Os resultados obtidos a partir da aplicação do questionário ELF nas 30 crianças pelos pais/responsáveis nas diferentes pontuações (pontuação total no silêncio, pontuação total na presença de ruído, pontuação total no silêncio + ruído, número de sim para atividades em volume fraco, número de sim para atividades em volume normal e número de sim para atividades em volume alto/forte, no silêncio e na presença de ruído), encontram-se na Tabela 1.

\section{DISCUSSÃO}

$O$ resultado estatisticamente não significante entre os escores obtidos nos questionários aplicados pelos pais e responsáveis de diferentes níveis econômicos e grau de instrução indica que o ELF é de fácil compreensão, demonstrando ser um instrumento valioso como medida de habilidade auditiva de crianças. Ressalta-se que a diferença encontrada apenas no item "atividades sonoras em volume normal" pode ter sido uma ocorrência ao acaso, devendo ser novamente investigado em grupos maiores e no grupo com deficiência auditiva.

A avaliação da confiabilidade segundo os diferentes níveis de escolaridade é importante no contexto da aplicação de um questionário para auto-preenchimento, à medida que o grau de escolaridade pode afetar a compreensão da pergunta e, consequentemente, a resposta ${ }^{(22)}$.

Os modelos tradicionais de avaliação audiológica usam protocolos de testes com tecnologia avançada, mas não abordam questões de suporte para o envolvimento familiar, ou seja, criam poucas oportunidades para a família ${ }^{(23)}$. Tais modelos não deixam de ser eficientes para a detecção precoce da deficiência auditiva, mas os pais geralmente não reagem positivamente ao diagnóstico por participarem apenas de forma passiva durante o processo ${ }^{(23)}$. O ELF vem sendo indicado como um modelo de questionário para acompanhamento audiológico centrado na família, e é descrito como uma ponte para os pais entre a formalidade dos testes e o desempenho diário do seu filho em casa $^{(23)}$. Existem poucos estudos evidenciando como o papel dos pais pode refletir positivamente no desenvolvimento da criança, mas é consenso entre os pesquisadores que a atuação dos pais é crítica no processo terapêutico ${ }^{(24)}$.

Dessa forma, o instrumento ELF, traduzido e adaptado para o idioma Português, pode auxiliar os pais a melhor entender e participar dos aspectos relacionados à deficiência auditiva de seus filhos. Este questionário tem como objetivo prover essa interação crianças-pais, com os mesmos participando ativamente do processo diagnóstico/terapêutico. De fato, estudos quantitativos que utilizam questionários com perguntas fechadas procuram aproximar-se de uma dada realidade ${ }^{(25)}$, ou seja, as questões são formuladas para serem utilizadas como modelos que são apenas indicadores de uma determinada condição ou evento que se pretende avaliar ${ }^{(22)}$.

Uma base fundamental da intervenção centrada na família é o compromisso de ajudar a mesma a entender ações realizadas em período precoce, identificar as melhores formas para os pais aprenderem a lidar com as novas informações que irão receber, bem como a adotar estratégias para serem usadas em casa com seu filho. Este tipo de proposta estabelece uma relação importante com os familiares. Através desta relação, os membros da família descobrem os seus trunfos naturais e o que precisam fazer para facilitar o crescimento da criança deficiente auditiva ${ }^{(26)}$, como, por exemplo, no ELF, identificar qual a distância ideal de escuta para a criança no silêncio e no ruído, para sons em diferentes intensidades.

O ELF poderá contribuir para o aprimoramento de protocolos de avaliação da percepção da fala em crianças brasileiras deficientes auditivas de zero a três anos ${ }^{(27)}$, uma vez que a escassez de instrumentos formais e objetivos comercialmente disponíveis e indicados para avaliação e diagnóstico, na área da Fonoaudiologia é significativa. Ressalta-se que os reais objetivos de um processo de avaliação só podem ser atingidos quando instrumentos e procedimentos adequados são utilizados ${ }^{(27)}$. O ELF propõe um modelo de acompanhamento audiológico centrado na família, visto que a intervenção precoce inclui não só a família mas também as interações com vários profissionais, e a visão dos pais como parceiros na equipe ${ }^{(1)}$. 
São sugeridas novas investigações com o ELF com crianças deficientes auditivas.

\section{CONCLUSÃO}

O instrumento ELF foi traduzido e adaptado culturalmente para a população brasileira, mantendo a sigla ELF na versão em português. Houve equivalência cultural entre as versões do ELF nas línguas inglesa e portuguesa uma vez que $100 \%$ dos pais não manifestaram dificuldades de interpretação e compreensão das questões elaboradas e dos termos utilizados.

O estudo permitiu verificar sua confiabilidade para observação e acompanhamento das etapas iniciais do comportamento auditivo.

\begin{abstract}
Purpose: To translate and adapt the Early Listening Function (ELF) questionnaire into the Brazilian Portuguese language, and to evaluate the reliability of the test. Methods: It was carried out the translation of the ELF questionnaire into Brazilian Portuguese, the review of grammatical and idiomatic equivalents (reversed translations), and linguistic and cultural adaptation. Moreover, the intra-researcher reproducibility was evaluated. After the translation, the ELF was carried out with 30 children between zero and three years old, with no history of hearing loss risk, lack of family complaint regarding the overall development of the child, and no indicators of hearing loss disability. The questionnaire was applied in 12 cases of hearing detection at different distances, in quiet and noisy environments. Results: Results were analyzed using a descriptive statistical study based on the results obtained on the ELF score sheet. Conclusion: The ELF questionnaire was translated and culturally adapted for the studied population. In Brazilian Portuguese, its designation maintained the acronym ELF. The study verified the instrument's reliability for observing and monitoring the initial stages of hearing behavior.
\end{abstract}

Keywords: Translations; Hearing disorders/diagnosis; Hearing tests; Questionnaires; Hearing loss/diagnosis; Early diagnosis; Infant; Language development

\title{
REFERÊNCIAS
}

1. Martin ST, Martin LG, Pedersen HF. A collaborative approach to fitting amplification. AudiologyOnline [Internet]. 2001 [cited 2008 Aug 28]. Available from: http://www.audiologyonline.com/articles/article_detail. asp?article_id $=298$

2. Carney AE, Moeller MP. Treatment efficacy: hearing loss in children. J Speech Lang Hear Res. 1998;41:S61-S84.

3. Anderson K. Parent involvement: the magic ingredient in successful child outcomes. The Hearing Review [Internet]. 2002 Nov [cited 2007 Nov 28]. 9(11):24-7. Available from:_http://www.hearingreview.com/ issues/articles/2002-11_02.asp?

4. Gravel JS, O'Gara J. Communication options for children with hearing loss. Ment Retard Dev Disabil Res Rev. 2003;9(4):243-51.

5. Canho PGM, Neme CMB, Yamada MO. A vivência do pai no processo de reabilitação da criança com deficiência auditiva. Estud Psicol (Campinas). 2006;23(3):261-9.

6. Fitzpatrick E, Coyle DE, Durieux-Smith A, Graham ID, Angus DE, Gaboury I. Parents' preferences for services for children with hearing loss: a conjoint analysis study. Ear Hear. 2007;28(6):842-9.

7. Novaes BCAC, Balieiro CR. Terapia fonoaudiológica da criança surda. In: Ferreira LP, Befi-Lopes DM, Limongi SCO, organizadores. Tratado de fonoaudiologia. São Paulo: Roca; 2004. p.732-9.

8. Moret ALM, Freitas VS, Ferreira MCF, Alvarenga KF, Bevilacqua MC. Curso para pais de crianças deficientes auditivas: estudo do conhecimento dos pais em um módulo intermediário. Distúrb Comun. 2007;19(1):25-37.

9. Motti TFG, Pardo MBL. Diagnóstico da deficiência auditiva: compreensão dos pais e seguimento às orientações. Acta ORL [Internet]. 2003 [citado 2007 Nov 28]; 21(2):1-15. Disponível em: http://www. actaorl.com.br/detalhe_artigo.asp?id=14

10. Delgado-Pinheiro EMC, Castiquini EAT, Lopes AC, Bevilacqua MC. Parâmetros considerados nos procedimentos de avaliação da percepção dos sons da fala. Pró-Fono. 2003;15(3):317-24.

11. Castiquini EAT. Escala de integração auditiva significativa: procedimento adaptado para a avaliação da percepção da fala [dissertação]. São Paulo: Pontifícia Universidade Católica; 1998.

12. Geers AE. Techniques for assessing auditory speech perception and lipreading enhancement in young deaf children. Volta Rev. 1994;96(5):85-96.

13. Orlandi ACL, Bevilacqua MC. Deficiência auditiva profunda nos primeiros anos de vida: procedimento para a avaliação da percepção da fala. Pró-Fono. 1998;10(2):87-91.

14. Delgado EMC, Bevilacqua MC. Lista de palavras como procedimento de avaliação da percepção dos sons da fala para crianças deficientes auditivas. Pró Fono. 1999;11(1):59-64.

15. Castiquini EAT, Bevilacqua MC. Escala de integração auditiva significativa: procedimento adaptado para a avaliação da percepção da fala. Rev Soc Bras Fonoaudiol. 2000;4(6):51-60.

16. McCracken W, Young A, Tattersall H. Universal newborn hearing screening: parental reflections on very early audiological management. Ear Hear. 2008;29(1):54-64.

17. Anderson KH. ELF - Early Listening Function: Discovery tool for parents and caregivers of infants and toddlers [Internet]. Design by Phonak Hearing system. [cited 2010 May 24]. Available from: http:// www.kandersonaudconsulting.com/uploads/ELF_Questionnaire.pdf

18. World Health Organization. Prevention of blindness and deafness. Grades of hearing impairment [Internet]. 2008 [cited 2008 Out 30]; Available from: http://www.who.int/pbd/deafness/hearing_impairment_ grades/en/index.html

19. Grupo IBOPE. Associação Nacional de Empresas de Pesquisa. Levantamento Sócio-Econônimo. IBOPE; 2000.

20. Guillemin F, Bombardier C, Beaton D. Cross-cultural adaptation of health-related quality of life measures: literature review and proposed guidelines. J Clin Epidemiol. 1993;46(12):1417-32. 
21. Ferreira PEA, Cunha F, Onishi ET, Branco-Barreiro FCA, Ganança FF. Tinnitus handicap inventory: adaptação cultural para o português brasileiro. Pró-Fono. 2005;17(3):303-10.

22. Lopes CS, Faerstein E. Confiabilidade do relato de eventos de vida estressantes em um questionário autopreenchido: estudo Pró-Saúde. Rev Bras Psiquiatr. 2001;23(3):126-33.

23. Gravel JS, McCaughey CC. Family-centered audiologic assessment for infants and young children with hearing loss. Semin Hear. 2004;25(4):309-18.
24. Kurtzer-White E, Luterman D. Families and children with hearing loss: grief and coping. Ment Retard Dev Disabil Res Rev. 2003;9(4):232-5.

25. Tyler RS, Perreau AE, Ji H. Validation of the Spatial Hearing Questionnaire. Ear Hear. 2009;30(4):466-74.

26. Brown AS. The art and science of home visits. ASHA Leader. 2005; 15: 6-7.

27. Giusti E; Befi-Lopes DM. Tradução e adaptação transcultural de instrumentos estrangeiros para o Português Brasileiro (PB). Pró-Fono. 2008;20(3):207-10.

Anexo 1. ELF traduzido e adaptado para o idioma Português Brasileiro

\begin{tabular}{|c|c|c|c|c|c|c|c|c|}
\hline ELF & $15 \mathrm{~cm}$ & $1 \mathrm{~m}$ & $2 \mathrm{~m}$ & $3 \mathrm{~m}$ & $\begin{array}{c}\text { Sala } \\
\text { vizinha } \\
(+5 \mathrm{~m})\end{array}$ & $\begin{array}{l}\mathrm{N}^{\circ} \mathrm{de} \\
\operatorname{sim}\end{array}$ & $\begin{array}{l}\mathrm{N}^{\circ} \mathrm{de} \\
\text { talvez }\end{array}$ & $\begin{array}{l}\text { Menor distância que a } \\
\text { criança respondeu ao } \\
\text { barulho }\end{array}$ \\
\hline \multicolumn{9}{|l|}{ Atividades sonoras em volume fraco } \\
\hline \multicolumn{9}{|l|}{ 1. A mãe dizendo "ch, ch, ch" bem baixinho } \\
\hline \multicolumn{9}{|l|}{$\begin{array}{l}\text { 2. Esfregando as palmas rapidamente com } \\
\text { as mãos juntas }\end{array}$} \\
\hline \multicolumn{9}{|l|}{ 3. Estalo de língua fraquinho } \\
\hline \multicolumn{9}{|l|}{ 4. A mãe dizendo "bu, bu, bu" bem baixinho } \\
\hline \multicolumn{9}{|l|}{ Atividades sonoras em volume normal } \\
\hline \multicolumn{9}{|l|}{$\begin{array}{l}\text { 5. Abrir toda a água da torneira (ex: pia da } \\
\text { cozinha ou do banheiro) }\end{array}$} \\
\hline \multicolumn{9}{|l|}{$\begin{array}{l}\text { 6. A mãe cantando uma canção (ex. } \\
\text { "Parabéns pra você") }\end{array}$} \\
\hline \multicolumn{9}{|l|}{ 7. Batida de palmas fraca } \\
\hline \multicolumn{9}{|l|}{$\begin{array}{l}\text { 8. O pai falando "xip, xip, xip" em tom normal } \\
\text { de voz }\end{array}$} \\
\hline \multicolumn{9}{|l|}{ Atividades sonoras em volume forte/alto } \\
\hline \multicolumn{9}{|l|}{ 9. O pai falando "xuxa,xuxa" em voz alta } \\
\hline \multicolumn{9}{|l|}{$\begin{array}{l}\text { 10. Batida forte na porta, com as mãos } \\
\text { fechadas }\end{array}$} \\
\hline \multicolumn{9}{|l|}{$\begin{array}{l}\text { 11. Segurar duas colheres viradas, uma } \\
\text { para a outra, e batê-las com força na palma } \\
\text { da sua mão, duas vezes }\end{array}$} \\
\hline $\begin{array}{l}\text { 12. Bater numa frigideira ou panela com } \\
\text { uma colher de metal ou madeira }\end{array}$ & & & & & & & & \\
\hline
\end{tabular}

Traduzido de: Anderson KH. ELF - Early Listening Function: Discovery tool for parents and caregivers of infants and toddlers [Internet]. Design by Phonak Hearing System. [cited 2010 May 24]. Available from: http://www.kandersonaudconsulting.com/uploads/ELF_Questionnaire.pdf 
Anexo 2. Folha de registro das pontuações do ELF

Atividades em ambiente silencioso e ambiente com ruído

( $S$ - sim / A - às vezes)

\begin{tabular}{|c|c|c|c|c|c|c|c|c|c|c|c|c|c|}
\hline & \multicolumn{4}{|c|}{ Fraco } & \multicolumn{4}{|c|}{ Normal } & \multicolumn{4}{|c|}{ Alto/Forte } & \multirow{2}{*}{$\begin{array}{c}\text { Total (100 } \\
\text { possíveis) }\end{array}$} \\
\hline & $\begin{array}{c}\mathrm{N}^{\circ} \mathrm{de} \\
\mathrm{S}\end{array}$ & $\begin{array}{c}\text { Multiplicar } \\
\text { x12 }\end{array}$ & $\begin{array}{c}N^{\circ} \text { de } \\
A\end{array}$ & $\begin{array}{c}\text { Multiplicar } \\
\text { x10 }\end{array}$ & $\begin{array}{c}N^{\circ} \text { de } \\
S\end{array}$ & $\begin{array}{c}\text { Multiplicar } \\
\text { x8 }\end{array}$ & $\begin{array}{c}N^{\circ} \text { de } \\
A\end{array}$ & $\begin{array}{c}\text { Multiplicar } \\
\text { x6 }\end{array}$ & $\begin{array}{c}\mathrm{N}^{\circ} \text { de } \\
\mathrm{S}\end{array}$ & $\begin{array}{c}\text { Multiplicar } \\
\text { x5 }\end{array}$ & $\begin{array}{c}N^{\circ} \text { de } \\
A\end{array}$ & $\begin{array}{c}\text { Multiplicar } \\
\text { x3 }\end{array}$ & \\
\hline \multicolumn{14}{|l|}{$15 \mathrm{~cm}$} \\
\hline \multicolumn{14}{|l|}{$1,0 \mathrm{~m}$} \\
\hline \multicolumn{14}{|l|}{$2,0 \mathrm{~m}$} \\
\hline \multicolumn{14}{|l|}{$3,0 \mathrm{~m}$} \\
\hline $\begin{array}{l}\text { Sala } \\
\text { vizinha }\end{array}$ & & & & & & & & & & & & & \\
\hline
\end{tabular}

Pontuação Total

\begin{tabular}{|c|c|c|c|c|c|c|c|}
\hline Distância & $\begin{array}{l}\text { Pontuação total } \\
\text { em ambiente } \\
\text { silencioso }\end{array}$ & $\begin{array}{l}\text { Pontuação } \\
\text { total no silêncio } \\
\text { +ruído }=(\mathrm{S}+\mathrm{R})\end{array}$ & $\begin{array}{c}\mathrm{N}^{\circ} \text { de } \\
\text { respostas } \mathrm{S} \\
\text { para atividades } \\
\text { em volume } \\
\text { fraco }(\mathrm{S}+\mathrm{R})\end{array}$ & $\begin{array}{c}\mathrm{N}^{\circ} \text { de } \\
\text { respostas } \mathrm{S} \\
\text { para atividades } \\
\text { em volume } \\
\text { normal }(\mathrm{S}+\mathrm{R})\end{array}$ & $\begin{array}{l}\mathrm{N}^{\circ} \text { de respostas } \\
\mathrm{S} \text { para } \\
\text { atividades em } \\
\text { volume alto/ } \\
\text { forte }(\mathrm{S}+\mathrm{R})\end{array}$ & $\begin{array}{l}\text { Pontuação total } \\
\text { em ambiente } \\
\text { com ruído }\end{array}$ & $\begin{array}{l}\text { Audiologista } \\
\text { envolvido } \\
\text { na data do } \\
\text { trabalho } \\
\text { (iniciais) }\end{array}$ \\
\hline \multicolumn{8}{|l|}{$15 \mathrm{~cm}$} \\
\hline \multicolumn{8}{|l|}{$1,0 \mathrm{~m}$} \\
\hline \multicolumn{8}{|l|}{$2,0 \mathrm{~m}$} \\
\hline \multicolumn{8}{|l|}{$3,0 \mathrm{~m}$} \\
\hline Sala vizinha & & & & & & & \\
\hline
\end{tabular}

Traduzido de: Anderson KH. ELF - Early Listening Function: Discovery tool for parents and caregivers of infants and toddlers [Internet]. Design by Phonak Hearing System. [cited 2010 May 24]. Available from: http://www.kandersonaudconsulting.com/uploads/ELF_Questionnaire.pdf 